\title{
THERMODYNAMICAL PROPERTIES FOR WEAKLY INTERACTING DIPOLAR GASES WITHIN CANONICAL ENSEMBLES
}

\author{
K. GLAUM ${ }^{1}$ and H. KLEINERT ${ }^{2}$ \\ Institut für Theoretische Physik, Freie \\ Universität Berlin, 14195 Berlin, Germany \\ ${ }^{1}$ E-mail: glaum@physik.fu-berlin.de, ${ }^{2}$ E-mail: kleinert@physik.fu-berlin.de
}

\section{A. PELSTER}

Fachbereich Physik, Universität

Duisburg-Essen, 47048 Duisburg, Germany

E-mail:axel.pelster@uni-duisburg-essen.de

\begin{abstract}
We set up a recursion relation for the partition function of a fixed number of harmonically confined bosons. For an ideal Bose gas this leads to the wellknown results for the temperature dependence of the specific heat and the ground-state occupancy. Due to the diluteness of the gas, we include both the isotropic contact interaction and the anisotropic dipole-dipole interaction by an infinite-bubble sum of the lowest-order perturbative results. Due to the anisotropy of the dipole-dipole interaction, the thermodynamic quantities of interest crucially depend on the trap configuration.
\end{abstract}

Keywords: Dipolar gas; Bose-Einstein condensation; Canonical ensemble.

\section{Many-Body Path Integral Formalism}

The aim of this work is to give a path-integral description for a system of a fixed particle number $N$. To this end, we start with the imaginary-time evolution amplitude which is given by the following $N$-fold path integral

$$
\begin{aligned}
\left(\boldsymbol{x}_{1}, \ldots, \boldsymbol{x}_{N} ; \tau_{b}\right. & \left.\mid \boldsymbol{x}_{1}^{\prime}, \ldots, \boldsymbol{x}_{N}^{\prime} ; \tau_{a}\right)^{B} \equiv \frac{1}{N !} \sum_{P} \prod_{n=1}^{N}\left[\int_{\boldsymbol{x}_{n}\left(\tau_{a}\right)=\boldsymbol{x}_{n}^{\prime}}^{\boldsymbol{x}_{n}\left(\tau_{b}\right)=\boldsymbol{x}_{P(n)}} \mathcal{D}^{3} x_{n}(\tau)\right] \\
& \times \exp \left\{-\frac{1}{\hbar}\left(\mathcal{A}^{(0)}\left[\boldsymbol{x}_{1}, \ldots, \boldsymbol{x}_{N}\right]+\mathcal{A}^{(\mathrm{int})}\left[\boldsymbol{x}_{1}, \ldots, \boldsymbol{x}_{N}\right]\right)\right\}
\end{aligned}
$$

Therein we have to sum over all possible $N$-particle orbits which begin at $\left(\boldsymbol{x}_{1}^{\prime}, \ldots, \boldsymbol{x}_{N}^{\prime}\right)$ and go to $\left(\boldsymbol{x}_{1}, \ldots, \boldsymbol{x}_{N}\right)$ during the imaginary-time interval 
$\left[\tau_{a}, \tau_{b}\right]$. All these orbits are weighted with the help of the euclidean action which contains the interaction-free part

$$
\mathcal{A}^{(0)}\left[\boldsymbol{x}_{1}, \ldots, \boldsymbol{x}_{N}\right] \equiv \sum_{n=1}^{N} \int_{\tau_{a}}^{\tau_{b}} d \tau\left[\frac{M}{2} \dot{\boldsymbol{x}}_{n}^{2}(\tau)+V\left(\boldsymbol{x}_{n}(\tau)\right)\right]
$$

and the interaction

$$
\mathcal{A}^{(\mathrm{int})}\left[\boldsymbol{x}_{1}, \ldots, \boldsymbol{x}_{N}\right] \equiv \frac{1}{2} \sum_{n, m=1}^{N} \int_{\tau_{a}}^{\tau_{b}} d \tau V^{(\mathrm{int})}\left(\boldsymbol{x}_{n}(\tau)-\boldsymbol{x}_{m}(\tau)\right)
$$

Here, $M$ is the particle mass, $V$ denotes the harmonic background potential

$$
V(\boldsymbol{x})=\frac{M}{2}\left(\omega_{x}^{2} x^{2}+\omega_{y}^{2} y^{2}+\omega_{z}^{2} z^{2}\right),
$$

$V^{(\text {int) }}$ stands for the interaction potential which is specified later on, and the prime over the sum in (3) indicates that any self interaction is avoided. Since we deal with indistinguishable bosons, the evolution amplitude (1) contains a sum over all $N$ ! possible permutations $P$. Furthermore, for describing the thermodynamics of the $N$-particle ensemble, we have to calculate its partition function

$$
Z_{N}^{B}(\beta) \equiv \int d^{3} x_{1} \ldots d^{3} x_{N}\left(\boldsymbol{x}_{1}, \ldots, \boldsymbol{x}_{N} ; \hbar \beta \mid \boldsymbol{x}_{1}, \ldots, \boldsymbol{x}_{N} ; 0\right)^{B},
$$

where only the $\hbar \beta$-periodical path configurations in (1) contribute. Thereby, $\beta \equiv 1 / k_{B} T$ represents the reciprocal temperature. The requirement of indistinguishability of bosons complicates further calculations considerably even for the ideal Bose gas, as is explicitly shown in the next section.

\section{Canonical Ensemble for Ideal Particles}

Here, we discuss the situation for a non-interacting $N$-boson system and omit therefore the interaction (3). For this case, the action is given by (2) as the sum of $N$ single-particle actions, so that our $N$-particle evolution amplitude factorizes into $N$ single-particle ones. This yields for the partition function (5)

$Z_{N}^{(0) B}(\beta)=\frac{1}{N !} \sum_{P} \int d^{3} x_{1} \ldots d^{3} x_{N}\left(\boldsymbol{x}_{P(1)} ; \hbar \beta \mid \boldsymbol{x}_{1} ; 0\right)^{(0)} \ldots\left(\boldsymbol{x}_{P(N)} ; \hbar \beta \mid \boldsymbol{x}_{N} ; 0\right)^{(0)} .(6)$

Because of the occurrence of non-trivial permutations single-particle amplitudes are not necessarily periodical and one is led to multiple cycles as represented in Fig. 1. In general, such an $n$-cycle is defined by

$$
h_{n}(\beta) \equiv \int d^{3} x_{1} \ldots d^{3} x_{n}\left(\boldsymbol{x}_{1} ; \hbar \beta \mid \boldsymbol{x}_{n} ; 0\right)^{(0)} \ldots\left(\boldsymbol{x}_{2} ; \hbar \beta \mid \boldsymbol{x}_{1} ; 0\right)^{(0)}=Z_{1}(n \beta) .
$$



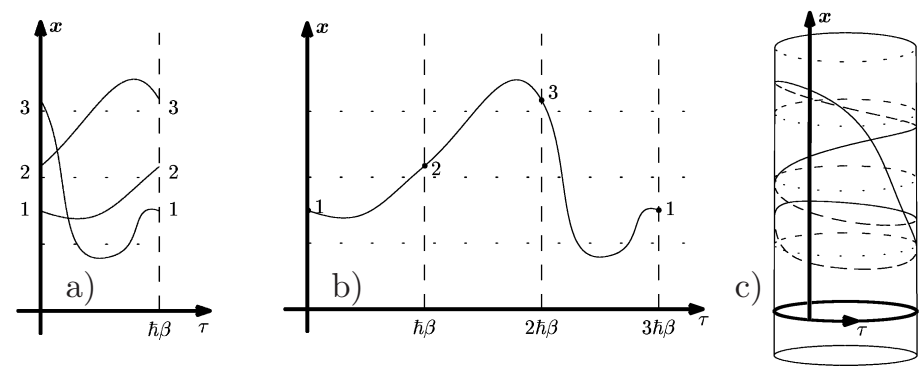

Fig. 1. Example of paths contributing to (6) for a cycle of length $n=3$ : a) final points coincide with initial points of the other particles, b) and c) show the same situation in an extended zone scheme and wrapped upon a cylinder.

The last equation follows from the imaginary-time translationary invariance of a single-particle amplitude and its group properties. This shows that the contribution of an $n$-cycle is simply the partition function of a single particle with a temperature lowered by a factor $n$. The full bosonical $N$-particle partition function (6) can be decomposed in such multiple cycles. The only problem is that the cycle structure strongly depends on the given permutation. One needs to know explicitly all possible cycle numbers with respect to the constraint of the fixed particle number $N$. In praxis, this becomes problematical for larger $N$ 's. But, according to Refs. 1,2, the partition function also follows from the recursion relation

$$
Z_{N}^{(0) B}(\beta)=\frac{1}{N} \sum_{n=1}^{N} Z_{1}(n \beta) Z_{N-n}^{(0) B}(\beta) \quad \text { with } \quad Z_{1}(n \beta)=\sum_{\boldsymbol{k}} e^{-\beta E_{\boldsymbol{k}}} .
$$

The partition function in vacuum $Z_{0}^{(0) B}(\beta)=1$ serves here for the starting point. We apply, furthermore, the results (8) for the heat capacity

$$
C_{N}^{(0) B}=k_{B} T \frac{\partial^{2}}{\partial T^{2}}\left\{T \ln Z_{N}^{(0) B}\left(1 / k_{B} T\right)\right\}
$$

and the ground-state occupancy, which is the probability for a particle being in the ground state $E_{\mathbf{0}}{ }^{2}$

$$
w_{N}^{(0) B}=\frac{1}{N} \sum_{n=1}^{N} e^{-n \beta E_{\mathbf{0}}} \frac{Z_{N-n}^{(0) B}(\beta)}{Z_{N}^{(0) B}(\beta)} .
$$

The results in an isotropic harmonic trap (4) are plotted for different particle numbers $N$ in Fig. 2. 

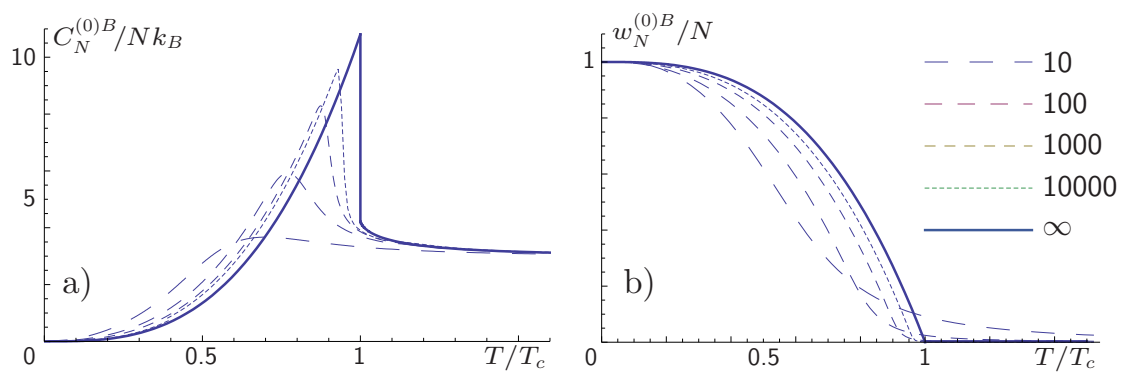

Fig. 2. a) Specific heat capacity and b) ground-state occupancy for an ideal Bose gas in an isotropic harmonic trap for different particle numbers $N$ versus reduced temperature. Here $T_{c} \equiv \hbar \omega[N / \zeta(3)]^{1 / 3} / k_{B}$ is the critical temperature in the thermodynamic limit.

\section{Dipolar Interacting System}

Now we describe the effect of an interaction upon thermodynamical properties of a Bose gas within the canonical ensemble theory. The system to be described is typically a gas of ${ }^{52} \mathrm{Cr}$ atoms which has recently been condensed in Stuttgart in T. Pfau's group. ${ }^{3}$ The interaction between the atoms can be modelled by a contact s-wave scattering plus a dipolar interaction

$$
V^{(\mathrm{int})}(\boldsymbol{x})=\frac{4 \pi \hbar^{2} a_{s}}{M} \delta(\boldsymbol{x})+\frac{\mu_{0} m^{2}}{4 \pi}\left(\frac{1}{|\boldsymbol{x}|^{3}}-\frac{3 z^{2}}{|\boldsymbol{x}|^{5}}\right)
$$

the latter being caused by the large magnetic dipole moment of these atoms, $m=6 \mu_{\text {Bohr }}$. In the experiment, the gas is trapped in a harmonic potential of a general form (4) with two almost equal frequencies giving rise to a cylindrically symmetric configuration. For aligned dipole moments two different configurations are possible. In the first configuration (I), the dipoles sit on top of each other and experience an attraction. In the second configuration (II), they are placed side by side and repel each other. The dipole forces are expected to distort slightly the condensate whose main interparticle forces come from an $s$-wave repulsion which is independent of the trap orientation. In Refs. 4,5, we have calculated the shift of the Bose-Einstein condensation temperatures caused by the dipolar forces. Here we discuss the thermodynamic properties of the anisotropic system at the entire low-temperature regime.

We begin with the full $N$-particle evolution amplitude (1) and Taylor expand the interaction factor $\exp \left\{-\mathcal{A}^{(\mathrm{int})} / \hbar\right\}$ around the zeroth order result of Section 2. The first-order contribution to the partition function (5) 
has the following cycle decomposition:

$$
Z_{N}^{(1) B}(\beta)=-\frac{1}{2 \hbar} \sum_{k=2}^{N} \sum_{l=1}^{k-1}\left[I_{l, k-l}^{(D)}(\beta)+I_{l, k-l}^{(E)}(\beta)\right] Z_{N-n}^{(0) B}(\beta) .
$$

Thereby many non-interacting cycles (7) occur which are combined to the non-interacting part $Z_{N-n}^{(0) B}$. Furthermore two different kinds of interacting cycles are always present. These are the direct and the exchange contributions, in contrast to the interaction-free one from Fig. $1 \mathrm{c}$ ) represented by

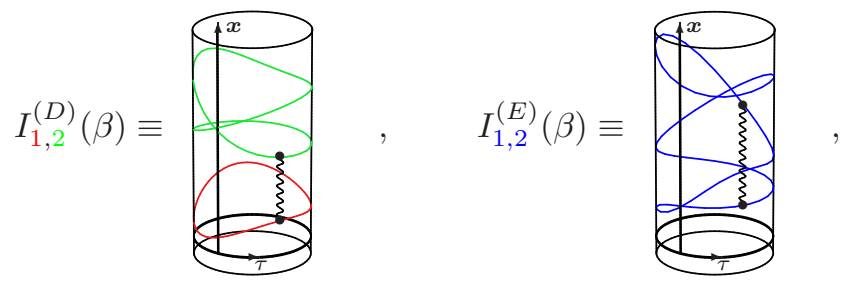

where the wiggly line stands for the interaction. Their simplified pictures represent the Hartree- and the Fock-like Feynman diagrams

$$
I_{1,2}^{(D)} \equiv{ }_{(1)} \bigcirc(2) \quad, \quad I_{1,2}^{(E)} \equiv \overbrace{(2)}^{(1)} .
$$

Combining both partition functions (8) and (12) to $Z_{N}^{(2)}=Z_{N}^{(0) B}+Z_{N}^{(0) B}+\ldots$ and performing the cumulant resummation one obtains the following new recursion relation for the full partition function

$$
Z_{N}^{B}(\beta)=\frac{1}{N} \sum_{n=1}^{N}\left\{Z_{1}(n \beta)-\frac{n}{\hbar} \sum_{l=1}^{n-1}\left[I_{l, n-l}^{(D)}(\beta)+I_{l, n-l}^{(E)}(\beta)\right]+\ldots\right\} Z_{N-n}^{B}(\beta) .
$$

Calculating both interacting contributions yields the proportionality $I^{(D),(E)} \propto \beta$. Hence, for low temperatures, the second term in the curly brackets of (14) is much larger than the first interaction-free summand leading to negative full partition function. The perturbative result must therefore be resummed. This can be done self-consistently by using the renormalized cycle contributions $\tilde{Z}_{1}(n \beta)=e^{-\beta n \tilde{E}_{k}^{(n)}}$ instead of the interaction-free terms $Z_{1}(n \beta)$, where

$$
\tilde{E}_{\boldsymbol{k}}^{(n)}=E_{\boldsymbol{k}}-\left[\Sigma_{n}^{(D)}(\boldsymbol{k})+\Sigma_{n}^{(E)}(\boldsymbol{k})\right] / \hbar \beta
$$

are new energy levels shifted by the self-energies. They are represented by Feynman diagrams related to (13) by cutting one line:

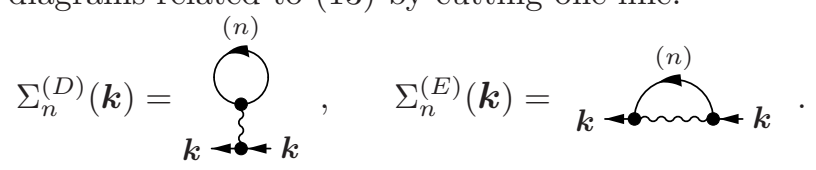



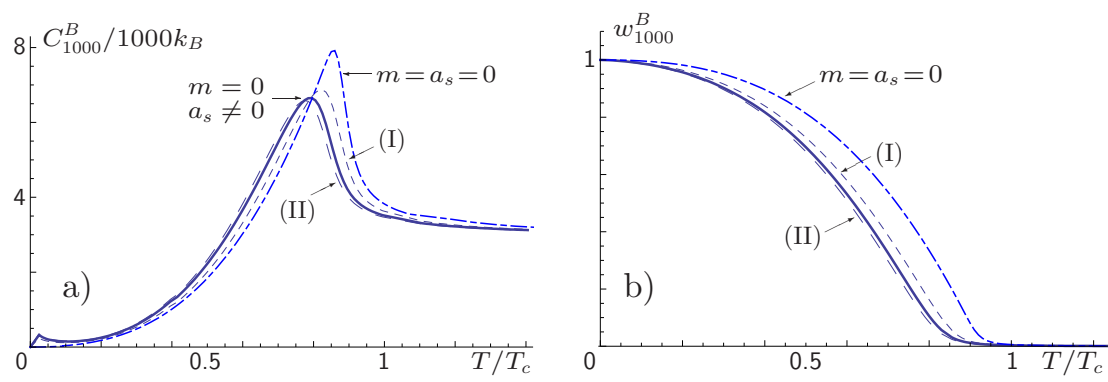

Fig. 3. a) Specific heat capacity and b) ground-state occupancy for $N=1000$ ideal (dashed-dotted curves), contact (solid curves) and dipolar interacting (dashed curves) bosons within a cylindrically symmetric harmonic trap. The configurations (I) or (II) correspond to dipoles which sit on each other or are placed side by side, respectively.

All interaction terms are given by functions of the form

$$
\Sigma_{n}^{(D, E)}(\boldsymbol{k}), I_{l, n-l}^{(D, E)} \sim \hbar \beta\left\{\frac{4 \pi \hbar^{2} a_{s}}{M}-\frac{\mu_{0} m^{2}}{3} f\left(\kappa_{l, n}^{(D, E)}\left[\omega_{\perp}, \omega_{\|}\right]\right)\right\}
$$

with certain anisotropy parameters $\kappa_{l, n}^{(D, E)}\left[\omega_{\perp}, \omega_{\|}\right]$, which are smaller than 1 for prolate configurations and larger than 1 for oblate ones. The first term in the curly brackets of (17) represents the contribution of the contact interaction irrespective of the trap anisotropy. The second term corresponds to the shift of the dipolar interaction and crucially depends on the anisotropy. The configurations (I) and (II) are described by the anisotropy functions

$$
f^{(\mathrm{I})}(\kappa)=-2 f^{(\mathrm{II})}(\kappa)=\frac{2 \kappa+1}{1-\kappa}-\frac{3 \kappa}{(1-\kappa)^{3 / 2}} \operatorname{Artanh} \sqrt{1-\kappa} .
$$

We apply these results to calculate the canonical $N$-particle full partition function. From this, the specific heat capacity and the ground-state occupancy for $N$ interacting particles have been obtained by analogy with the non-interacting case. The results for $N=1000$ are presented in Fig. 3.

\section{References}

1. F. Brosens, J. T. Devreese, and L. F. Lemmens, Phys. Rev. E 55, 227 (1997).

2. K. Glaum, H. Kleinert, and A. Pelster, Phys. Rev. A 76, 063604 (2007).

3. A. Griesmaier, J. Werner, S. Hensler, J. Stuhler, and T. Pfau, Phys. Rev. Lett. 94, 160401 (2005).

4. K. Glaum, A. Pelster, H. Kleinert, and T. Pfau, Phys. Rev. Lett. 98, 080407 (2007).

5. K. Glaum and A. Pelster, Phys. Rev. A 76, 023604 (2007). 